\title{
Performance of Microstrip Patch Antenna on a Reinforced Carbon Fiber Composite Ground Plane
}

\author{
A. Mehdipour, A.-R. Sebak, C. W. Trueman, I. D. Rosca and S. V. Hoa \\ A. Mehdipour, A.-R. Sebak and C. W. Trueman (Department of Electrical and \\ Computer Engineering, Concordia University, Montreal, QC, Canada) \\ Email: a_meh@ece.concordia.ca \\ I. D. Rosca and S. V. Hoa (Department of Mechanical and Industrial Engineering,
} Concordia University, Montreal, QC, Canada)

The performance of a microstrip patch antenna operating against a carbon fiber composite ground plane is investigated and compared with results for a copper ground, both numerically and experimentally. An electromagnetic (EM) model of the composite antenna is developed using Microwave Studio for numerical analysis. The ground plane is made of reinforced continuous carbon fiber (RCCF) composite, which has anisotropic conductivity. The direction of the fibers in the ground plane affects the current distribution, and hence the impedance bandwidth and the matching characteristics of the antenna.

Introduction: Advanced carbon-fiber composite (CFC) materials are being used widely in the avionic and automobile industry as a good replacement for metal because of their higher strength, lower weight, and lower cost [1, 2]. CFCs have lower electrical conductivity than metals, and so the shielding effectiveness is of concern when electromagnetic compatibility (EMC) must be maintained. Reinforced continuous carbon-fiber (RCCF) composite is used extensively due to its low cost and ease of fabrication [2]. The high oxidation stability allows RCCF composites to be used for applications where metals would suffer corrosion. Very recently, we explored the use of RCCF to build radio frequency identification (RFID) antennas [3] and wideband antennas [4]. 
There are many antennas mounted on or integrated to the surface of the mobile vehicles for different communication systems, radar, navigation, surveillance and so on. Showing low level of drag and being suitable for conformal applications, planar microstrip antennas are a very good candidate to be installed on such vehicles [5]. In many cases, the body of vehicle is used as the ground plane for the antennas. Therefore, investigating the performance of such antennas operating on a composite ground plane is of interest [6].

In this letter, we investigate the performance of a metal patch antenna operating on a ground plane made of RCCF composite material, both numerically and experimentally. In RCCF materials the carbon fibers run in one direction only, and so the conductivity is anisotropic, being high along the direction of fibers, but low in the perpendicular direction [2]. The effect of fibers direction on the impedance bandwidth of the antenna is studied. Due to the lower conductivity of composite material compared to the metals, the antenna with composite ground plane is more lossy, leading to lower radiation efficiency. The gain and radiation pattern of the antenna with composite ground plane is evaluated and also compared to that of antenna with copper ground plane.

RCCF Composite Material: Fig. 1 shows a typical RCCF composite material. The carbon fibers embedded in epoxy resin are oriented in a specific direction. The effective complex permittivity of one-layer composite depends on the thickness of layer $(t)$, fibers diameter $(D)$, the separation distance between fibers $(s)$ and the complex permittivity of the fibers and the host medium $\left(\varepsilon_{f}, \varepsilon_{m}\right)$. The effective complex anisotropic permittivity of the homogenized model of RCCF is given by [2]

$$
\begin{gathered}
\varepsilon_{y^{\prime}}^{-1}=(1-k) \varepsilon_{m}^{-1}+k \varepsilon_{f}^{-1} \\
\varepsilon_{x^{\prime}}=\varepsilon_{z^{\prime}}=(1-k) \varepsilon_{m}+k \varepsilon_{f}
\end{gathered}
$$

where $k$ is a coefficient which depends on the volume fraction of fibers inside host medium $\left(k=\pi D^{2} / 4 s t\right)$. In reality, the average diameter of fibers $(D)$ is about $7 \mu \mathrm{m}$ 
and separation distance between fibers is typically around $1 \mu \mathrm{m}$. The conductivity of RCCF is high along the direction of fibers $\left(\sigma_{x^{\prime}}\right)$, but low in the perpendicular direction $\left(\sigma_{y^{\prime}}\right)$ so that $\sigma_{x^{\prime}} / \sigma_{y^{\prime}}>>1$. Sheets of RCCF composite material are produced at the Concordia Center for Composites (CONCOM) [7]. The unidirectional carbon fiber prepreg (MTM 45-1), produced by Advanced Composites Group is cut to dimensions after thawing it for $24 \mathrm{~h}$ at room temperature. Then the composite is vacuum-bagged and cured in an autoclave for $4 \mathrm{~h}$ at $140^{\circ} \mathrm{C}$ under $40 \mathrm{psi}$ of pressure. In order to extract the permittivity tensor of the RCCF samples to be used in antenna simulations, standard waveguide setups are used for characterization as described in $[3,8]$.

Patch Antenna Grounded by RCCF Composite: Fig. 2 (a) shows the EM model of a microstrip patch antenna developed in MWS [9], operating in a frequency band centered at $5.6 \mathrm{GHz}$. The antenna is fed through a 50 -ohm SMA connector from the bottom of the ground plane. The square patch $(W=16.5 \mathrm{~mm})$ is printed on $\mathrm{RT} /$ Duroid 5880 substrate $\left(\varepsilon_{r}=2.2\right)$ with thickness of $1.5 \mathrm{~mm}$. The size of substrate is $L_{1} \times L_{2}=25 \times 28 \mathrm{~mm}^{2}$. Other geometrical parameters are $d=12.5$, and $g=7.25 \mathrm{~mm}$. The ground plane of the antenna is a $0.8 \mathrm{~mm}$-thick 4-layer RCCF composite material where the direction of carbon fibers in each layer is equally oriented by $\alpha$ degree with respect to $x$-axis. The composite material is characterized by permittivity tensor obtained using a standard G-band waveguide setup. The size of the RCCF ground plane is $D_{1} \times D_{2}=60 \times 60 \mathrm{~mm}^{2}$. The effective conductivity of the RCCF composite over the desired frequency range is $1500 \mathrm{~S} / \mathrm{m}$ along fiber direction and $15 \mathrm{~S} / \mathrm{m}$ perpendicular to the fibers. The effective relative permittivity is about 6 for both directions, hence is not anisotropic.

The reflection coefficient of the antenna with copper and RCCF ground plane is shown in Fig. 2 (b). It is observed that the resonant behavior of the antenna is taken away by rotating fibers toward so that they are parallel to the $y$-axis $\left(\alpha=90^{\circ}\right)$ the antenna is no longer resonant. The reason can be explained by the current 
distribution on the ground plane. In Fig. 3, the surface current distribution of the copper ground plane is compared with that of RCCF for $\alpha=0^{\circ}$ and $\alpha=90^{\circ}$. It is observed that the $x$-directed fibers support a current distribution quite similar to that on the copper ground, leading to similar resonant behavior and impedance matching. However, for $y$-directed RCCF ground plane $\left(\alpha=90^{\circ}\right)$, the current is forced to flow in $y$-direction, leading to a current distribution completely different from that in copper ground, and to poor impedance matching.

The patch antennas with copper and RCCF $\left(\alpha=0^{\circ}\right)$ ground plane were fabricated for measurement. An HP8720 network analyzer was used to measure the reflection coefficient as shown in Fig. 4. Good agreement is observed between measured and simulated results.

The normalized radiation pattern in both $H$ - and E-planes at the frequency of resonance, $5.6 \mathrm{GHz}$, is shown in Fig. 5 . It is observed that using RCCF composite ground plane instead of the copper ground changes the radiation pattern very little. However, the ohimc loss of RCCF composite reduces the gain and radiation efficiency. The peak gain of the antenna with RCCF ground plane is measured at resonant frequency and reported in Table 1. It is observed that the gain drops by about $3 \mathrm{~dB}$ when RCCF ground plane is used.

Conclusion: The performance of a microstrip patch antenna with ground plane made of reinforced continuous carbon fiber (RCCF) composite is investigated. The direction of the fibers in the ground plane plays an important role on the antenna performance. When the fibers are oriented in the direction of current flow in the copper ground plane, the RCCF ground plane can support the same current distribution as the copper ground plane. The patch antenna with the RCCF ground shows the same resonant behavior as the antenna with the copper ground plane. The radiation pattern of the antenna with RCCF ground is found to be almost the same as that of the antenna with copper ground. However, due to the higher loss of the RCCF composite compared to copper, the gain of the antenna over composite ground is 
about $3 \mathrm{~dB}$ smaller.

Acknowledgement: The authors would like to thank Natural Sciences and Engineering Research Council of Canada (NSERC) for supporting this work.

\section{References}

[1] De Rosa, I. M., Sarasini, F., Sarto, M. S., and Tamburrano, A., "EMC impact of advanced carbon fiber/carbon nanotube reinforced composites for next-generation aerospace applications," IEEE Trans. Electromagn. Compat., 2008, 50, (3), pp. $556-563$

[2] Holloway, C. L., Sarto, M. S., and Johansson, M., "Analyzing carbon-Fiber composite materials with equivalent-layer models," IEEE Trans. on Electromagn. Compat., 2005. 47, (4), pp. 833-844

[3] Mehdipour, A., Rosca, I. D., Sebak, A.-R., Trueman, C. W., and Hoa, S. V. "Advanced carbon-fiber composite materials for RFID tag antenna applications", Applied Computational Electromagnetic Society (ACES) Journal, 2010, 25, (3), pp. $218-229$

[4] Mehdipour, A., Sebak, A.-R., Trueman, C. W., Rosca, I. D., and Hoa, S. V., "Reinforced continuous carbon-fiber composites using multi-wall carbon nanotubes for wideband antenna applications," IEEE Trans. on Antenna and Propagat., 2010, 58, (7), pp. 2451-2456

[5] Josefsson, L., and Persson, P.: 'Conformal array antenna theory and design' (Wiley -IEEE Press, 2006.)

[6] Kiang, J.-F., "Rectangular patch resonator with laminated ground plane," IEEE Trans. on Antenna and Propagat., 1995, 43, (12), pp. 1361-1368

[7] CONCORDIA CENTER FOR COMPOSITES (CONCOM), Concordia University, QC, Canada, 1979. Online: http://concom.encs.concordia.ca

[8] Challa R. K., Kajfez D., Demir V., Gladden J. R., and Elsherbeni A. Z., "Characterization of multiwalled carbon nanotube (MWCNT) composites in a waveguide of square cross section," IEEE Microwave and Wireless Components 
Lett., 2008, 18, (3), pp. 161-163

[9] CST - Microwave Studio, Computer Simulation Technology, 2010. 
Table captions:

Table I : Peak gain of the patch antenna

Figure captions:

Fig. 1 (a) RCCF composite, (b) Cross-sectional view, (c) Optical micrograph of RCCF composite (scale bar $50 \mu \mathrm{m}$ )

Fig. 2 Microstrip patch antenna grounded by RCCF, (a) antenna geometry, (b) $S_{11}$ of the patch antenna

Fig. 3 Surface current distribution on the ground plane of the patch antenna

Fig. 4 Reflection coefficient of the fabricated patch antennas

Fig. 5 Normalized radiation pattern of the patch antenna 
Table 1

\begin{tabular}{|c|c|c|}
\hline Type of Ground Plane & Simulation & Measurement \\
\hline RCCF $\left(\boldsymbol{\alpha}=\mathbf{0}^{\circ}\right)$ & $5.1 \mathrm{~dB}$ & $5.8 \mathrm{~dB}$ \\
\hline Copper & $8.23 \mathrm{~dB}$ & $8.58 \mathrm{~dB}$ \\
\hline
\end{tabular}


Figure 1

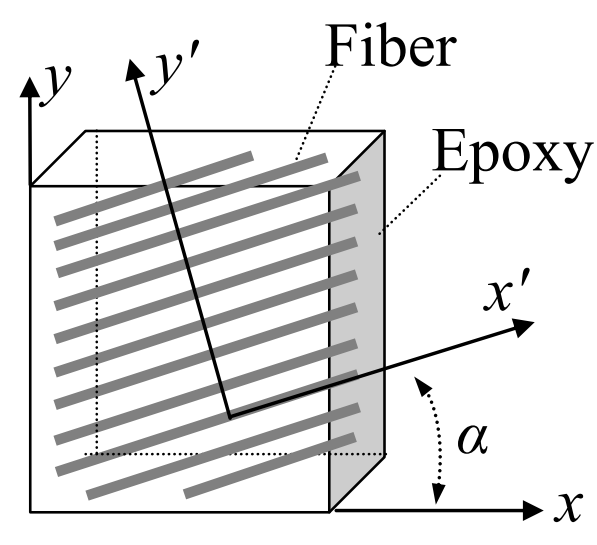

(a)

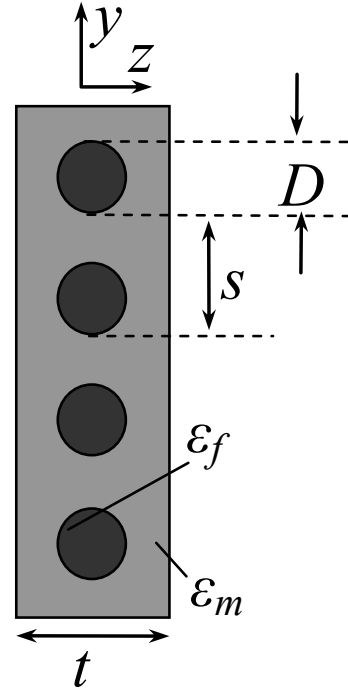

(b)

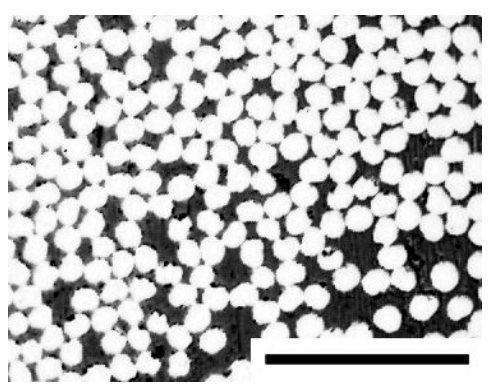

(c) 
Figure 2

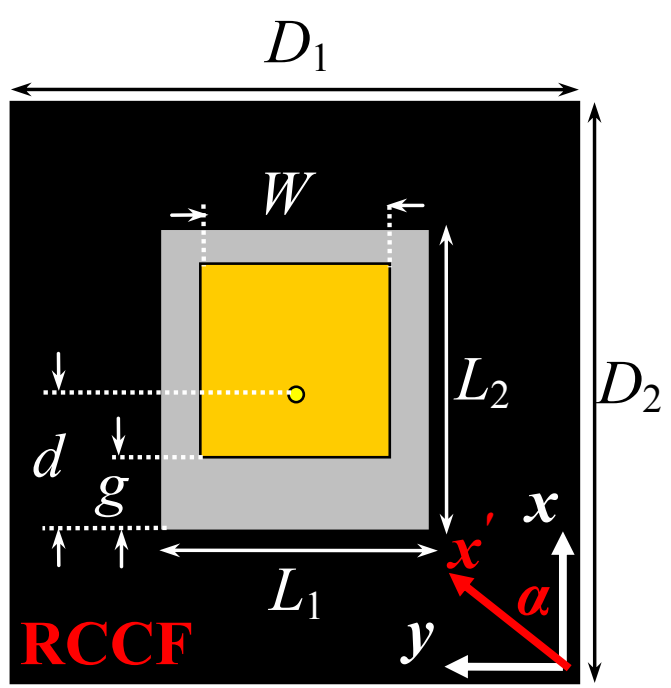

(a)

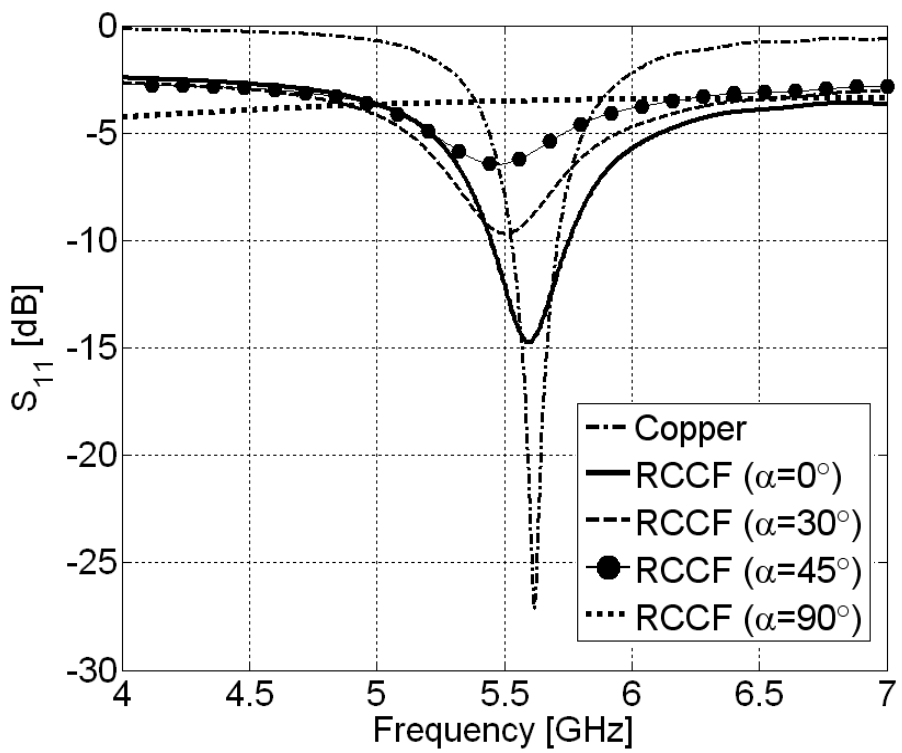

(b) 
Figure 3
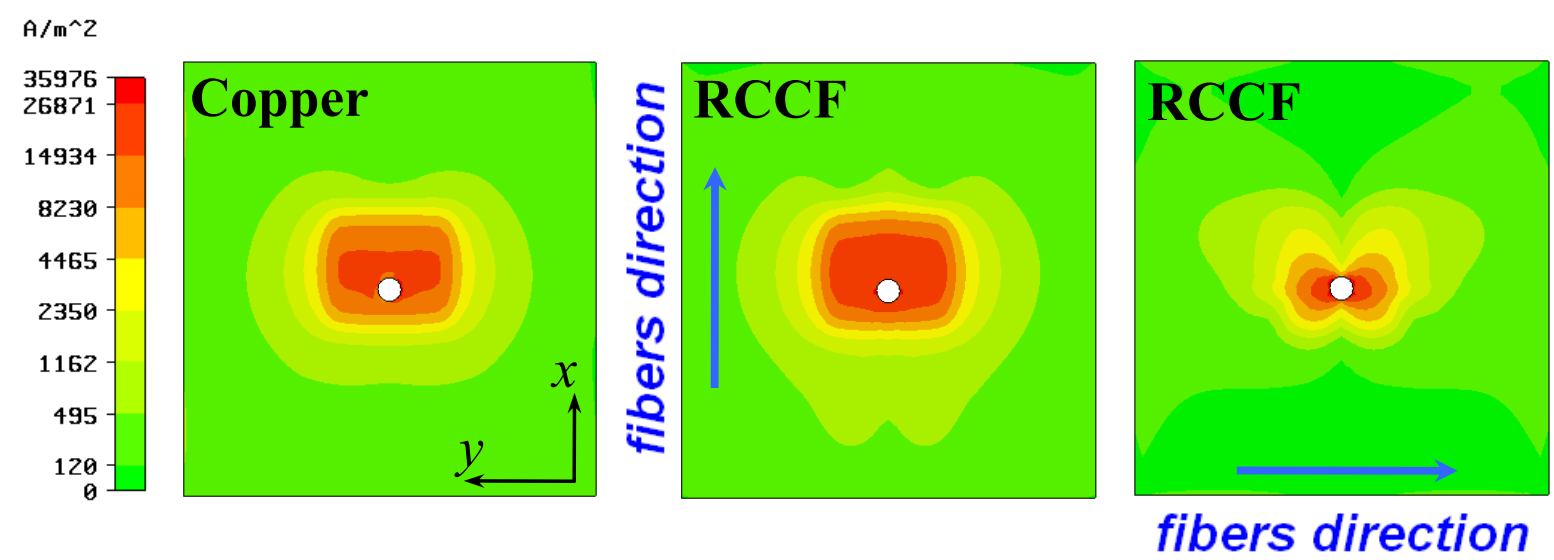
Figure 4

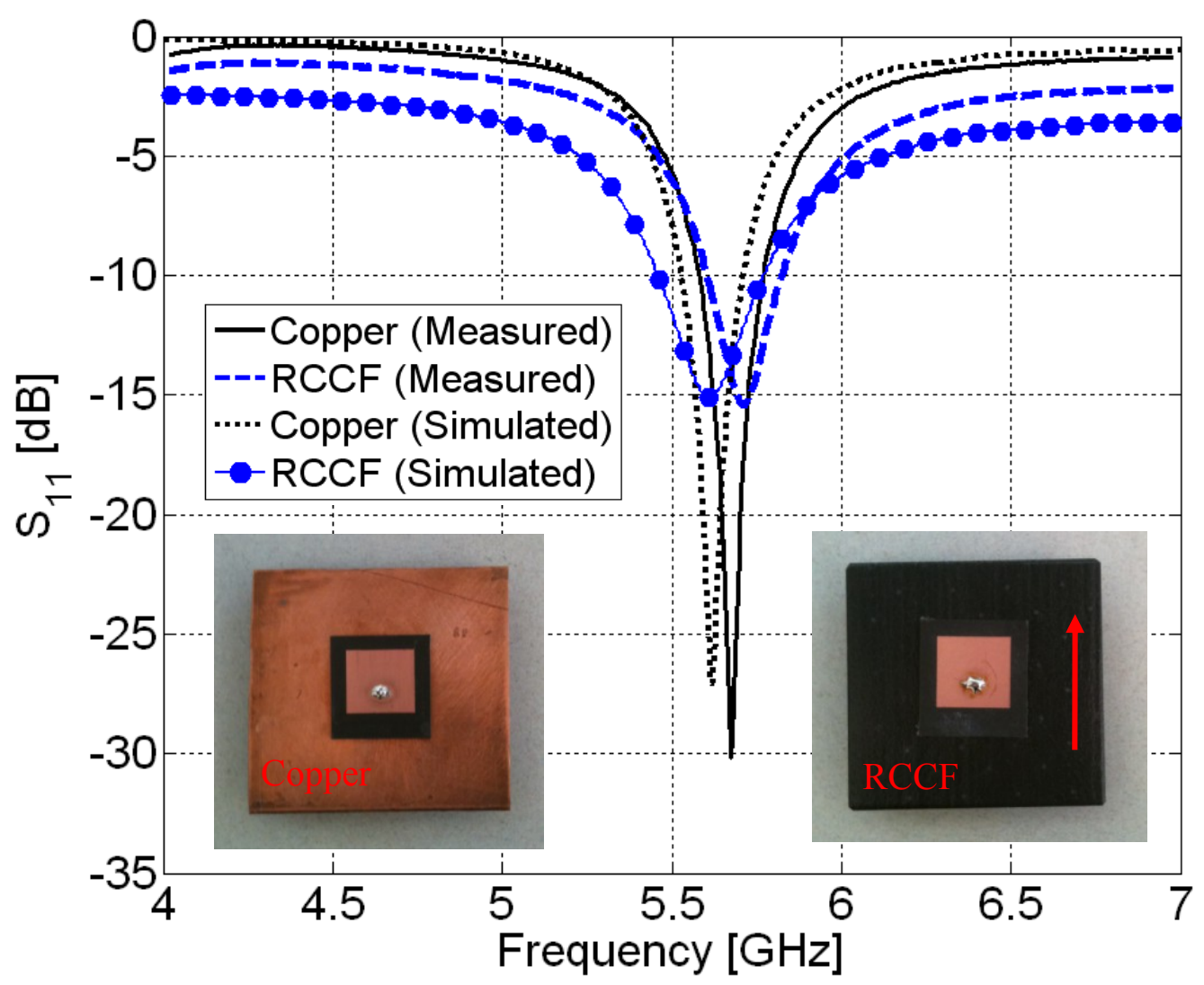


Figure 5

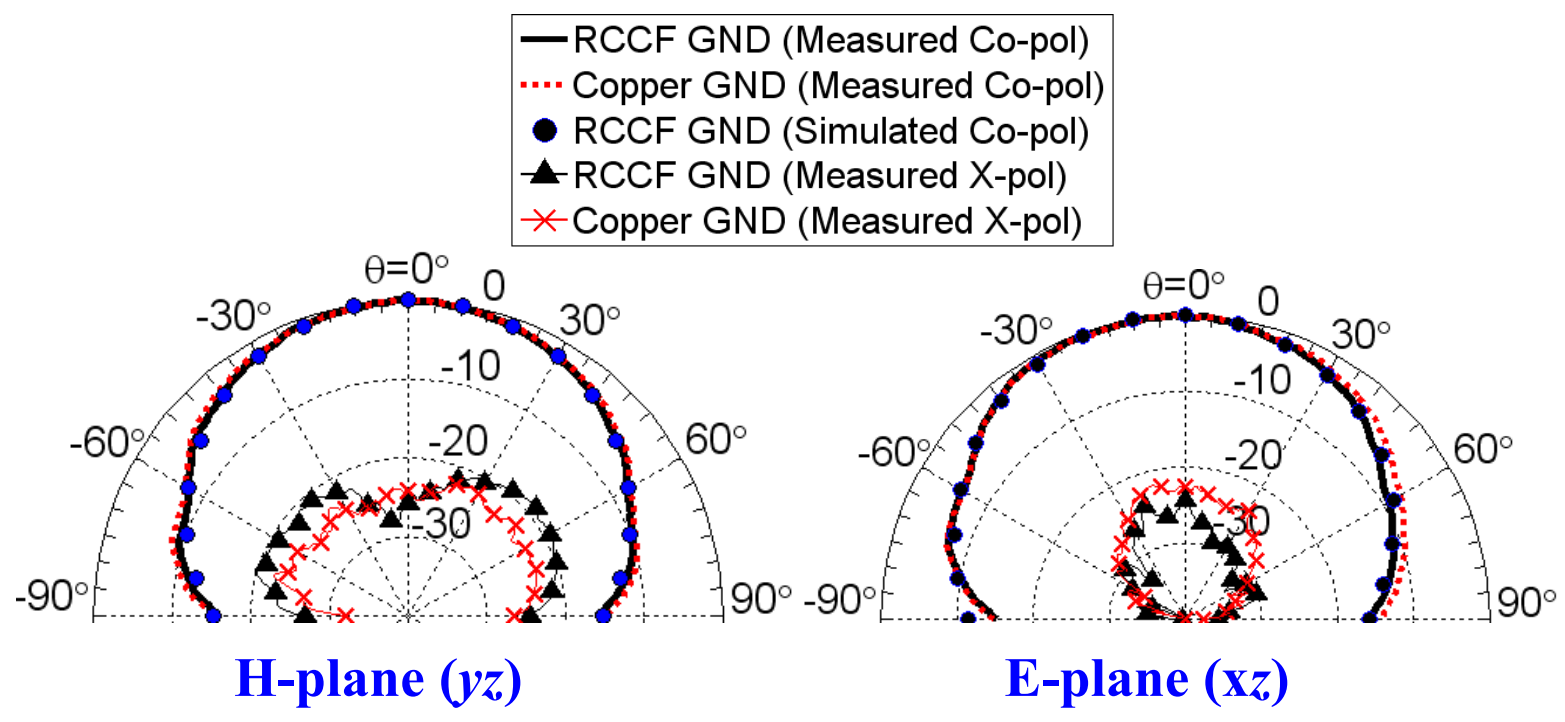

